\title{
MINI-REVIEW ON MALARIA AND HUMAN IMMUNODEFICIENCY VIRUS (HIV) IN SUB-SAHARA AFRICA
}

\author{
By \\ AHMAD MEGAHED A. SALEH ${ }^{1}$, MOSTAFA M. ELNAKIB ${ }^{1}$, \\ DENG MAYOM AROP MALEK ${ }^{2}$ And TOSSON A. MORSY ${ }^{3}$
} Military Medical Academy Cairo $11291^{1}$, South Sudan People's Defense Forces (SSPDF) ${ }^{2}$
And Faculty of Medicine, Ain Shams University, Cairo $11566^{3}$, Egypt

\begin{abstract}
HIV is the virus that causes HIV infection. AIDS is the most advanced stage of HIV infection. HIV is spread through contact with the blood, semen, pre-seminal fluid, rectal fluids, vaginal fluids, or breast milk of a person with HIV. In the United States, HIV is spread mainly by having anal or vaginal sex or sharing injection drug equipment, such as needles, with a person who has HIV. Meanwhile, Malaria is a life-threatening mosquito-borne blood disease caused by a Plasmodium parasite that infects a type of mosquito which feeds on humans. Once an infected mosquito bites a human, parasites multiply in the host's liver before infecting and destroying their red blood cells. Malaria patients are usually very sick with symptoms such as high fevers, shaking chills, and flu-like illness. Globally, WHO estimated that 212 million clinical cases of malaria occurred, and 429,000 people died of malaria, most of them children in Africa. Since many countries with malaria are already among the poorer nations, the disease maintains a vicious cycle of disease and poverty.
\end{abstract}

Key words: Sub-Sahara Africa, Malaria, human immunodeficiency virus, Mini-review

\section{Introduction}

Malaria and HIV infections often coexist in patients in many parts of the world due to geographic overlap of these two diseases. This is particularly true in the sub-Saharan Africa, where an estimated 40 million people are living with HIV and more than 350 million episodes of malaria occur yearly (Hewitt et al, 2006). There is also evidence of a negative interaction between these two infections. González et al. (2012) mentioned that HIV infection increases malaria susceptibility and reduces the efficacy of anti-malarial drugs. On the other hand, the effect of malaria on HIV-infected individuals has also been explored, with the parasitic infection increasing the risk of HIV disease progression and mother-to-child transmission of the HIV. Spread of malaria and parasitic resistance to anti-malarial could also be accelerated by the HIV-associated immunosuppression. Alemu et al. (2013) reported that the HIV infection increases the risk of the increase in the severity of malaria infection and burdens of malaria, which in turn facilitates rate of malaria transmission. Malaria infection is also associated with strong CD4+ cell activation and up-regulation of proinflamm- atory cytokines and it provides the ideal microenvironment for the spread of the virus among the CD4+ cells and for rapid HIV-1 replication. Also, malaria increases blood viral burden by different mechanisms. So, the high concentrations of HIV-1 RNA in blood were predictive of disease progression, and correlated with risk of the blood-borne, vertical, and virus sexual transmission.

\section{Review and Discussion}

Immunity to malaria is characterized by an age-related reduction in parasite burden, clinical symptoms, and prevalence of severe disease in individuals residing in an endemic area (Karp and Auwaerter, 2007). P. falciparum infection and the burden of parasitemia are often less severe in older adults than in children. Children are at increased risk since they have not yet acquired natural immunity; pregnant women transiently lose some of their acquired immunity due to the relative immunosuppression of pregnancy (Marodi, 2006). The degree of immunity is also related to transmission intensity, which varies geographically. HIV-related immunosuppression diminishes acquired immunity.

Clinical versus asymptomatic malaria: The individuals living in endemic areas might 
develop partial immunity to the disease following repeated infections. These "immune" individuals are not immune to infection per se. Following a bite by an infected mosquito, they will still develop parasitemia, but the severity of clinical symptoms is typically limited. This could affect estimates of clinical infection since patients who live in endemic areas were much less symptomatic and often without symptoms (Ogwang et al, 2018).

Physicians should be aware that malaria is not a clinical diagnosis but must be diagnosed, or excluded, by performing microscopic examination of blood films. The incubation period for $P$. falciparum infection is about 12 to 14 days. Longer incubation periods are more likely in semi-immune individuals and individuals taking ineffective malaria prophylaxis. The incubation period for $P$. vivax and $P$. ovale is also about two weeks; most relapses due to these species occur within three months. The incubation period for $P$. malariae is about 18 days; however, low grade asymptomatic infections can persist for years (Bartoloni and Zammarchi, 2012)

Impact of malaria on HIV: In vitro, malarIal antigens led to $\mathrm{T}$ cell activation and de novo productive HIV infection (Froebel et $a l, 2004)$. Episodes of malaria have been associated with declines in CD4 cell counts over time compared to HIV-infected patients without parasitemia (Mermin et al, 2006). In 190 French HIV-patients with imported malaria Mouala et al. (2008) found severe infection associated with a CD4 cell count $<350$ cells/microL $(\mathrm{OR}=2.58 ; 95 \%$ CI 1.2-5.6) .

Viral load: Malaria infection is associated with a temporary rise of HIV/RNA, although long term, this does not appear to hasten progression to AIDS (Hoffman et al, 1999). A cohort study performed in Malawi prospectively enrolled 348 aparasitemic patients to obtain the baseline HIV VL load and followed them over time with active surveyllance for malaria infection. A near doubling of HIV/RNA was demonstrated between baseline and follow-up in those patients who acquired malaria infection. Increases in viral load were greatest in patients with fever, CD4 counts $>300$ cells/microL and in those with a parasite density $>2000 /$ microL (Kublin et al, 2005). This rise was temporary, with HIV viral load returning to baseline at two months after treatment of malaria. If viral loads were used to monitor response to HAART, testing should be delayed if patient acquired a recent malaria infection. This transient increase in viral load would be predicted to have minimal impact on long term HIV progression, unless infections were frequent or remained occult without treatment. From a public health point, even a transient rise in HIV/RNA at a population level could impact sexual transmission (Crampin et al, 2002).

HIV-related survival: Comparing rates of HIV-related survival in malaria-endemic to non-endemic countries is problematic due to the higher prevalence of additional health care problems found in these geographic regions, which may also impact morbidity and mortality. In addition, there are few well-done studies in malaria-endemic areas to address this question. However, studies from Uganda and Malawi found a ten-year survival rate after HIV infection, which was comparable to rates reported in developed non-malaria endemic countries in the preHAART era (Jaffar et al, 2004).

Impact of HIV on malaria: Studies to define the impact of HIV on malaria have been conducted to examine various malaria disease markers including susceptibility, prevalence of malaria infection, the peripheral parasite burden, disease severity, and response to treatment.

Susceptibility: HIV serostatus and immunosuppression might be associated with an increased risk of susceptibility to malaria infection as reported in following studies: A cohort study was conducted in 224 HIV-infected and 125 HIV-uninfected adults in Malawi to assess whether HIV serostatus $t$ affected incidence of malaria (Patnaik et al, 2005). HIV-1 seropositivity was significantly associated with the first and second epi- 
sode of the parasitemia. The first risk of parasitemia episode was inversely related to the baseline CD4 cell count in HIV-1-seropositive persons. In Uganda, a retrospective study was performed in 1965 patients who received treatment for an episode of malaria (Kamya et al, 2006). Use of molecular genotyping showed that HIV-infected patients were at significantly higher risk of acquiring new infections than HIV-seronegative ones during the standard 28-day follow-up period.

In Zambia, a clinical trial on 971 adults with uncomplicated malaria; HIV infection was detected in $33 \%$ of them. Patients with CD4 counts $<300$ cells/microL were at sever risk for recurrent parasitemia, recrudescence, and new infection (Van Geertruyden $e t$ al, 2006).

Risk of clinical malaria: Host immunity, as measured by CD4 cell counts, appears to be a critical factor in the clinical presentation of malaria in HIV-infected patients. HIV status might also increase the risk of high-level parasitemia (French et al, 2001). In a longitudinal study of $600 \mathrm{HIV}$-infected patients in Malawi, malaria blood smears were examined monthly and were also performed if the patient had any illness suggestive of malaria (Laufer et al, 2006). Incidence of clinical malaria episodes was higher in patients with CD4 counts $<200$ cells/microL than in those with relatively preserved CD4 counts. Francesconi et al. (2001) reported that among 222 HIV-seropositive and 237 HIV-seronegative patients in Uganda who had routine and sick clinic visits, risk of clinical malaria was significantly higher in HIV-infected patients than in HIV-seronegative patients (16 vs. 10\%). This risk increased with advanced immunosuppression (six-fold higher in patients with a CD4 count $<200$ cells/microL compared to those with a CD4 count > 500 cells/microL). Parasitemia was more common in HIV-infected patients than HIVseronegative patients (12 vs. 6\%).

Severity of malaria: There were conflicting reports as to whether HIV serostatus contributed to the malaria severity (Muller, 1990).
This might be partly related to whether a patient lives in a region of stable or unstable malaria transmission (Butcher, 2005). The range of findings was reported: A prospective cohort study from South Africa examined the effect of HIV status and prior history of malaria on the clinical expression of malarial infection (Cohen et al, 2005). Non-immune HIV-infected patients with immunosuppression were significantly risky to have severe malaria (13/36 patients) than in nonimmune HIV-seronegative patients (9/75); odds ratio, 4.15 [95\% CI 1.57-10.97]. In 660 adults with HIV in Malawi followed over 12 years, only three had severe malaria with CD4 counts less than 200cells/mm3. Mouala et al. (2009) in France reported that using a hospital data-base, 104 HIV-infected patients \& $161 \mathrm{HIV}$-seronegative ones were identified who had acquired malaria during travel. HIV sero-status was the risk factor for severe malaria only in those patients who had a CD 4 cell count of $<350$ cells/microL.

Coinfection and spread of disease: A direct relationship has been noted between the height of plasma HIV/RNA levels and probability of transmission of HIV per coital act (Quinn et al, 2000). Since coinfection led to a near one-log increase in viral load in chronically HIV-infected patients, and HIV increases susceptibility to malaria infection, there are concerns that coinfection might be contributing to more spread of both diseases.

Using a mathematical model, authors estimated the impact of HIV and malaria on one another in Kenya, excess prevalence was $2.1 \%$ for HIV and $5.1 \%$ for malaria (AbuRaddad et al, 2006). Since 1980, this disease interaction might be responsible for 8500 excess HIV infections and 980,000 excess malaria episodes. Coinfection also facilitated the geographic expansion of malaria in areas where HIV prevalence was high. The prevalence of mosquito trnsmissible blood-borne forms (gametocytes), was also higher in the HIV-infected patients with immunosupprression (CD4 counts <200 cells/microL) that might have additional malaria implications 
transmission (Shah et al, 2996).

When malaria occurs during pregnancy, the disease is more severe, and hypoglycemia and respiratory complications are more common. Pregnant women residing in malaria endemic areas were highly susceptible to $P$. falciparum malaria, mainly during their first pregnancy, resulting in low birth weight babies and maternal anemia. This susceptibility was associated with placental sequestration of parasitized red blood cells expressing pregnancy-specific variant surface antigens. Acquisition of antibodies against these variant surface antigens might protect females and their offspring. Functions of such antibodies might include prevention of the placental sequestration or opsonisation of the parasitized cells for phagocytic clearance (Ataíde et al, 2010). Pregnancy-associated malaria is characterized by sequestration and multiplication of a distinct population of malarial parasites in the placenta, which expressed a specific class of variant surface antigens (VSAs). These antigens mediate adhesion of parasite-infected erythrocytes to chondroitin sulfate A (CSA) in intervillous space led to placental insufficiency. In each pregnancy, immunity to placental malaria occurred and maternal and fetal complications declined, associated with maternal production of antibody specific to CSA expressing parasites (Neres et al, 2008). This explained the clinical fact that better malariarelated birth outcomes occurred after multiple pregnancies. However, this immunologic benefit was lost in HIV-seropositive pregnant women as multiparous women have similar clinical outcomes to those of primigravidas (Bloland et al, 1995). In Malawi pregnant women with malaria from showed lower concentrations of CSA/VSA IgG in HIV-infected ones compared to those without HIV, and HIV infection impairs antimalarial immunity, especially responses to placental type VSA (Mount et al, 2004).

In Sub-Sahara, maternal health of HIVseronegative and seropositive pregnant women, dual infection with HIV and malaria was associated with an increased higher risk of parasitemias, maternal death, post-partum maternal anemia (Ayisi et al, 2003), and severe clinical disease compared to the HIVseronegative women (ter Kuile et al, 2004). Perinatal outcomes suggested that dual malaria infection and HIV led to increasing risk of adverse perinatal outcomes. A study to examine the relationship between maternal HIV infection, placental malaria of 2608 pregnant women, 138 (5\%) were HIV patients, neonatal mortality in infants born to mothers with both placental malaria and HIV infection was significantly greater than those born to mothers with either infection alone. The coinfection with malaria and HIV was associated with the increased risk of stillbirth (odds ratio 4.7, 95\% CI 1.34-16.78) and preterm delivery (odds ratio $=4.1,95 \%$ CI 2.17-7.75). Each infection was independently associated with an increased risk of low birth weight, low Apgar scores, and fetal growth restriction (Ticconi et al, 2004). They added that the malaria and HIV infections were independently associated with an increased risk of stillbirth, preterm delivery, low to very low birth weight, low Apgar scores, and fetal growth restriction. Dual infection with HIV and malaria was associated with an increased risk of maternal, perinatal, and early infant death.

Effect of malaria on HIV/RNA: Malarial infection results in a temporary increase in HIV/RNA, which returned to baseline after antimalarial treatment. However, an increase in viral load at the time of delivery might result in an increase in vertical transmission of HIV (Cao et al, 1997). During pregnancy, syncytio-trophoblast vesicles contributed to the maternal tolerance towards the fetus, but also to pathologies such as pre-eclampsia. Alterations in total and trophoblast microparticles associated with malaria and HIV in pregnant women might have an immunopathogenic role and the potential for placentalderived vesicles and microRNAs as the biomarkers of adverse outcomes during pregnancy and malaria (Moro et al, 2016). The 
most commonly used regimen for IPT in the pregnant women is sulfadoxine-pyrimethamine $(1500 \mathrm{mg}$ sulfadoxine \& $75 \mathrm{mg}$ pyrimethamine) administered at the specific intervals after "quickening" (mother's recognition of fetal movement). Standard dosing given in Africa was usually two treatment courses given in the $2^{\text {nd }} \& 3^{\text {rd }}$ trimesters as part of the routine scheduled antenatal clinic visits (Schultz et al, 1994). WHO (2004) recommended that pregnant women in sub-Saharan, Africa in areas of stable malaria transmission must receive the intermittent preventive antimalarial therapy (IPT) to reduce placental malaria and complications. But, information on optimal dosing for HIV-infected pregnant women is still not clear, although the bulk of the data suggested that greater reduction in peripheral and placental parasitemia and a significant increase in the mean birth weight were achieved with monthly IPT rather than the standard two-course regimens (Ter Kuile and Steketee, 2007). In an intense malaria area transmission in Kenya, monthly IPT was more efficacious in preventing placental malaria compared to a two-dose course among the HIV-infected women (25 vs. $7 \%$ ). A randomized non-blinded study compared the two doses of sulfadoxine-pyrimethamine vs. monthly sulfadoxine-pyrimethamine given during pregnancy in $266 \mathrm{HIV}-$ seropositive and $1626 \mathrm{HIV}$-seronegative ones in Malawi (Parise et al, 1998). In the HIV-seropositive cohort, the monthly regimen provided a significant reduction in the incidence of placental malaria compared to the two-dose arm (8 vs. 22\%). Data from a randomized controlled study of monthly versus two-dose SP therapy in HIV-seropositive women in Zambia confirmed a dose-dependent benefit of SP in reducing maternal anemia, peripheral and cord blood parasitemia, infant prematurity, and low birth weight. From a logistic viewpoint, many women who were randomized to two doses of SP actually only received one, which was far inferior in efficacy. Hamer et al. (2007) suggested that the monthly SP intermittent therapy may be a more effective strategy in minimizing the risk of inadvertent underdosing and poor out-comes. The incidence of malaria in that study was low, which was attributed to additional use of the safe insecticide-treated bed-nets

WHO (2004) recommended that HIVinfected pregnant women should receive at least three courses of IPT to achieve the same benefits that HIV-seronegative women appear to have with the standard two-dose regimen. In addition to defining the optimum dosing frequency, the amount of drug needed during pregnancy is still uncertain. A pharmacokinetic study found that the area under the curve (AUC) concentration for sulfadoxine was significantly shorter during pregnancy than in the postpartum state; however, HIV status itself had little influence on pharmacokinetic parameters in pregnant women (Green et al, 2007).

Malaria diagnosis: In many regions worldwide, malaria is diagnosed on the basis of fever alone and medications are administered without confirmatory testing. Consequently the diagnosis of malaria may be overestimated and inappropriately treated. Conversely, if a diagnostic workup is pursued, a positive malaria smear may be misinterpreted as a cause for fever in an HIVinfected patient (or HIV-negative), when an alternative etiology is present. Also, malaria infection and bacteremia may coexist in the same patient (Bronzanet al, 2007). Diagnosis of malaria should be considered in all febrile patients who have traveled to or lived in malaria-endemic areas, malaria smears in patients with fever, a careful history and physical examination was required to look for alternative causes of fever as typhoid (Kaplan et al, 2009). The differential diagnosis was a must when evaluating a patient with advanced immunosuppression (e.g., cryptococcal meningitis and tuberculous meningitis). If a patient's fever was not resolved after three days on antimalarial therapy, then an alternative etiology should be considered (Qu et al, 2017). 
Diagnosis of HIV: Robb et al. (2015) stated that acute human immunodeficiency virus type 1 (HIV-1) infection is a major contributor to transmission of HIV-1. An understanding of acute HIV-1 infection may be important in the development of treatment strategies to eradicate HIV-1 or achieve a functional cure. They concluded that the viral-load set point occurred at a median of 31 days after the first detection of plasma viremia and correlated with peak viremia. Few symptoms and signs were observed during acute HIV-1 infection, and they were most common before peak viremia. However, two important factors are important to keep in mind when considering diagnosis of HIV in malaria endemic areas: Coinfection with HIV and malaria is common. Travellers to and from areas where considerable overlap between high malaria transmission and elevated prevalence of HIV infection exists, augment the probability that returning travellers to non-endemic countries would present with both infections. The presence of such co-infection increased the severity of malaria episodes and also changed the HIV progression. Thus, the diagnosis of clinically apparent malaria infection in a patient from an endemic area should prompt routine HIV counseling and testing (Pereira et al, 2015).

Treatment of malaria: Kamya et al. (2006) stated that HIV infection increased the burden of malaria by increasing susceptibility to infection or by decreasing the response to antimalarial treatment. They added that the HIV-1 seroprevalence rate was surprisingly high in adults presented with malaria. This data supported the implementation of routine HIV counseling and testing for adults with uncomplicated falciparum malaria. HIV-1 infection increased the susceptibility to new malarial infections but did not increase the recrudescence risk in adults. Besides, many countries were experiencing downward trends in their HIV epidemics after 10-15 years of large global investment (Beyrer and Karim, 2013). However, some countries now face international donor withdrawal despite non-decreasing epidemics (Enserink, 2014). Shattock et al. (2017) in Kazakhstan stated that despite the non-decreasing HIV epidemic, the international donors were soon expected to withdraw funding this disease.

Efficacy: Conflicting results have been published as to whether coinfection with the HIV portends a poor treatment outcome: Harris et al. (2018) reported that Malawi successfully achieved Millennium Development Goal (MDG) four by decreasing the under-5 mortality rate by two-thirds in 2012 . Despite this progress child mortality was still high and in 2013, the leading causes of death in under-5s were malaria, acute respiratory infections \& HIV/AIDS. They added that, despite a significant reduction in the pediatric inpatient mortality in Malawi, but infectious diseases remains the predominant cause. However, the molecular genotyping assays in one study demonstrated that HIVinfected patients were more likely to have acquired new infections (Santoro and Perno, 2013). This suggested that the HIV-infected patients may have increased susceptibility to infection. A total of 508 adults in western Kenya were followed for 28 days after SP treatment for malaria. Treatment failure was higher HIV-seropositive patients with a CD4 count $<200$ cells/microL compared to those HIV-seronegative ones (21 vs. $8 \%$ ), with a higher rate of both the new infections and recrudescent infections in the HIV positive cohort (Byakika-Kibwika et al, 2007).

Malaria and HIV infection impose immense and overlapping burdens in sub-Saharan Africa. Prophylaxis with trimethoprimsulfamethoxazole and insecticide-treated bed nets reduce the incidence of malaria among HIV-infected children, but the protection is incomplete, and even with the use of these protective strategies, the burden of malaria remains great in areas of high-intensity transmission Treatment of malaria in HIVinfected patients is similar to treatment of HIV-uninfected individuals (Kamya et al, 2007). Management of patients with severe malaria presented a broad array of clinical 
challenges given the complex pathophysiology of infection involving multiple organ systems. These challenges increased many fold in the endemic areas where access to diagnostic and therapeutic tools might be limited. Death due to severe malaria could occur within hours of presentation, so prompt assessment and initiation of antimalarial therapy was essential. Patients should be evaluated with attention to find consistent with malaria as well as additional and /or alternative causes of presenting symptoms. For treatment of non-pregnant adults with severe malignant malaria intravenous artesunate was suggested and for children with severe malignant malaria intravenous quinine must be given. For treatment of pregnant women with severe malignant malaria in the $2^{\text {nd }} \& 3^{\text {rd }}$ trimesters intravenous artesunate was also suggested with total duration of artemisinin based therapy was 3 days (Rosenthal, 2008). For pregnant women with severe malignant malaria in the $1^{\text {st }}$ trimester intravenous quinine was suggested with quinine/quinidine for severe malaria was 7 days (Dondorp et al, 2005).

Drug interactions: Limited data exist on pharmacokinetic interactions between antiretroviral therapy and antimalarial drugs. A study was undertaken to evaluate potential interactions between the combinations of amodiaquine plus artesunate (AQ/AS) and HIV non-nucleoside reverse transcriptase inhibitor, efavirenz (German et al, 2007). The study was prematurely discontinued after the first two patients developed severe asymptomatic hepatotoxicity more than one month after study completion. No other etiology for the marked flare in aminotransferases was identified. Addition of efavirenz resulted in significant increases of amodiaquine serum concentrations in both patients (AUC increased by $115 \& 302 \%$, respectively). Optimal treatment regimens for HIV-infected patients are not clear due to limited data. Aminotransferases should be monitored in patients on regimens these medications.

Effect of ART on Plasmodium species: In vitro and animal data suggest that HIV protease inhibitors (PIs) exert antiplasmodial effects on erythrocytic-stage parasites (Lek-Uthai et al, 2008). In a rodent parasite model, lopinavir and saquinavir inhibited the preerythrocytic stage of parasite development. Therapeutic interventions directed at the preerythrocytic (intrahepatic) stage have the advantage of inhibiting the parasite while occurred in low numbers and before blood stream infection (Hobbs et al, 2009).

Current first-line ART regimens in the malaria-endemic areas usually include non-nucleoside reverse transcriptase inhibitors, rather than PIs. Clinical trials to evaluate the potential inhibitory effects of PIs on malaria infection have not yet been performed.

Malaria prophylaxis: Trimethoprimsulfame thoxazole is used for prophylaxis of opportunistic infections such as pneumocystis also has efficacy in the prevention of malaria. Authors evaluated effect of trimethoprimsulfamethoxazole on morbidity and mortality and malarial drug resistance.

Effect on morbidity and mortality: Prophylaxis with trimethoprim-sulfamethoxazole (cotrimoxazole) decreased the rates of morbidity and mortality from malaria in HIVinfected adults and children, even in areas with a high rate of malaria resistance (Mermin et al, 2006). The following observations illustrate the range of findings: In a prospective observational study, trimethoprimsulfamethoxazole prophylaxis was associated with a $46 \%$ reduction in mortality and lower rates of malaria, diarrhea, and hospital admissions in 509 HIV-infected patients followed over a two-year period compared to 1522 HIV-negative household controls. Another prospective study the evaluated 466 HIV-infected patients in Uganda evaluated the effect of trimethoprim-sulfamethoxazole prophylaxis, bed-nets, and ART on the acquisition of malaria. ART was specifically offered to those patients with a CD4 count < 250 cells/microL (Kamya et al, 2007). All the three interventions contributed to a significantly lower frequency of clinical disease 
compared to the period of observation alone. A direct relationship was noted between a lower risk of malaria and improvement in the CD4 cell count. These data are consistent with prior observations on the susceptibility of patients with advanced AIDS to malaria infection.

In contrast to lack of harm in resource-rich countries, discontinuation of trimethoprimsulfamethoxazole in patients attained a CD4 cell count $>200$ cells/microL may have a significant negative clinical impact in malariaendemic areas. A randomized-controlled trial that assessed the safety of discontinuing trimethoprim-sulfamethoxazole in 836 Ugandan patients was halted by the Data Safety Monitoring Board after 116 days (Campell et al, 2009). The patients assigned to discontinuation arm compared to those who continued prophylaxis had significantly increased rates of malaria ( $12 \%$ v vs. $0.4 \%) \&$ diarrhea $(25 \%$ vs. $14 \%)$. The trial was not designed to examine HIV progression rates during repeated episodes of malaria or antibiotic resistance with ongoing drug exposure.

Effect on drug resistance: The widespread use of trimethoprim-sulfamethoxazole in the malaria endemic countries as OI prophylaxis raises the concern of exacerbating antifolate drug-resistant malaria. In a vitro malaria models showed that antifolate resistance could arise quickly under drug pressure and the development of sulfadoxine-pyrimethamine resistance is observed commonly in many endemic areas. To address the effect of drug pressure on the development of parasite antifolate drug resistance mutations, a randomized, open-label study was conducted of trimethoprim-sulfamethoxazole prophylaxis for malaria. Such preliminary data provide some reassurance of the efficacy of trimethoprimsulfamethoxazole. In view of the large impact on HIV-related mortality and malaria morbidity and mortality, the use of trimethoprim-sulfamethoxazole prophylaxis must be considered in patients with CD4 counts < 200 cells/microL or with a history of recurrent malaria (Thera et al, 2005).
Nursing role: After acute stage of illness was treated with parenteral therapy and patient can swallow, a complete course of oral therapy must be administered. Preferably treatment given to patients in rural endemic areas with suspected severe malaria that can not begin immediately by intravenous therapy. Death due to severe malaria can occur within hours of admission, so prompt assessment and initiation of anti-malarial therapy are essential, and followed by concurrent supportive care to manage life-threatening risk: 1- Pulmonary complications as pulmonary edema, acute respiratory distress syndrome, and lower respiratory tract infection. Management requirements may range from supplementary oxygen to mechanical ventilation. 2- Neurologic complications include altered sensorium seizure and coma. Clinical evaluation includes full physical examination, calculation of Blantyre coma score, fundus examination and lumbar puncture. 3Hematologic complications include severe anemia and coagulopathy. Decisions regarding transfusion should be tailored to individual patient circumstances. 4- Hypoglycemia (blood glucose $<40 \mathrm{mg} / \mathrm{dL}$ or $<2.2 \mathrm{mmol} / \mathrm{L}$ ) is a common complication of malaria and a marker of severe disease; it must be suspected in any patient who suddenly deteriorates. Hypoglycemic patients must have intravenous access established promptly followed by given $50 \%$ dextrose $(1 \mathrm{~mL} / \mathrm{kg})$ with repeat blood glucose measurement after 15 minutes. 5- Hypovolemia must be assessed on an individual basis. Adults with malaria appear to be more vulnerable to fluid overload than children, with a thin line between underhydration (and thus worsening renal impairment) and overhydrating (and risk of pulmonary edema). But, incidence of new infections might be higher and recrudescence of drug resistant parasites may occur so careful follow-up was indicated. The HIV survived in the syringes for up to six weeks (Abdala et al, 2000).

On the other hand, nurses work along continuum of HIV care, from prevention and 
testing, engagement in care, sexual and reproductive health, long-term management through to palliative care. They work in a wide variety of environments and care for people with HIV across the lifespan (Johnstone and Crock, 2016). Thus, imperative that nurses 'have the knowledge, skills, and "right attitude" to be able to respond to the issues in an appropriate, ethically warranted and just manner (Tilley and Crock, 2016).

Malaria prevention: Use of insecticidetreated mosquito bed-nets (ITNs) for the prevention of malaria in endemic areas, such as Africa, is one of the main goals of the "Roll Back Malaria" partnership. However, the scaling-up of availability of such netting, particularly for children and pregnant women, has been slow (Miller et al, 2007).

General prevention: Arthropod-borne infectious diseases mainly mosquitoes are the major risk cause of morbidity and mortality among inhabitants and travellers to Sub-Sahara Africa (Braack et al, 2018). Obtaining a detailed travel itinerary and understanding traveler-specific and destination-specific risk factors can help mitigate diseases risks. DEET, Picaridin, PMD, and IR3535 are repellents that offer sufficient protection against arthropod bites. IR3535 does not provide adequate protection against Anopheles mosquitoes, and should not be used in malaria-endemic areas (Alpern et al, 2016). General protective measures, such as bite avoidance, protective clothing, insecticidetreated bed-nets, and the insecticide-treated clothing is a must. Spatial repellents could prevent nuisance biting, but did not prevent vector-borne disease (Morsy, 2012). Travel to Sub-Sahara must find health advice, vacc ines, and medicines at destination, and plan a visit with doctor at least before leave. Pack smart and prepare a travel health kit, c consider insurance that covers medical care and emergency evacuation (CDC, 2019).

\section{Conclusion and Recommendations}

There are five malarial species that infect humans. But, HIV and malaria coinfection most frequently occurs with $P$. falciparum, due to overlapping geographic distribution between this species and HIV individuals. Co-infection with malaria is associated with a transient increase in HIV/RNA. If viral loads are being used to monitor the response to HAART, testing should be delayed for approximately two months if the patient has had a recent malaria infection. HIV infection is associated with increased susceptibility, higher parasitemia, and an increased risk for recurrent malaria, particularly in patients with CD4 counts <200 cells/microL. Dual infection with HIV and malaria during pregnancy leads to adverse maternal and perinatal outcomes. Monthly sulfadoxine-pyrimethamine $(1500 \mathrm{mg}$ sulfadoxine \& $75 \mathrm{mg}$ pyrimethamine) throughout pregnancy, start in $2^{\text {nd }}$ trimester. HIV test in adults with clinical malaria in a region where natural immunity normally develops. Do an accurate diagnosis of malaria in the context of HIV coinfection is very difficult in endemic areas. An HIV-infected patient may have fever unrelated to malaria, even in setting of a positive blood smear with low-level parasitemia. This may lead to an incorrect diagnosis and inappropriate therapy. Malaria treatment in the HIV-infected patients similar to HIVuninfected ones, but, incidence of new infection might be higher and recrudescence of drug resistant parasites occurred, so careful follow-up. Trimethoprim-sulfamethoxazole (160mg trimethoprim and $800 \mathrm{mg}$ sulfamethoxazole daily) and bednets in HIV-infected patients with CD4 counts <200 cells /microL living in malarial endemic areas is indicated.

Trimethoprim-sulfamethoxazole may also be considered in patients with higher CD4 counts with history of recurrent malaria. Decision to institute TS prophylaxis against malaria for patients with CD4 >200 depend on transmission intensity, which varies by geography and season. ART decreases malaria incidence, and particular attention should be paid to administering ART to treatment candidates living in malaria-endemic areas.

\section{References}

Abdala, N, Reyes, R, Carney, JM, Heimer, R, 
2000: Survival of HIV-1 in syringes: Effects of temperature during storage. Subst. Use Misuse 35, 10:1369-83.

Abu-Raddad, LJ, Patnaik, P, Kublin, JG, 2006: Dual infection with HIV and malaria fuels the spread of both diseases in sub-Saharan Africa. Science 314:1603-6.

Alemu, A, Shiferaw, Y, Addis, Z, Mathewos, B, Birhan, W, 2013: Effect of malaria on HIV/ AIDS transmission and progression. Parasit Vectors Jan 17; 6:18. doi: 10.1186/1756-3305-6-18. Alpern, JD, Dunlop, SJ, Dolan, BJ, Stauffer, WM, Boulware, DR, 2016: Personal protection measures against mosquitoes, ticks, and other arthropods. Med. Clin. North. Am. 100, 2:30316

Ataíde, R, Hasang, W, Wilson, DW, Beeson, J G, et al, 2010: Using an improved phagocytosis assay to evaluate the effect of HIV on specific antibodies to pregnancy-associated malaria. PLoS One. May 25;5(5):e10807. doi: 10.1371/journal.pone.0010807.

Ayisi, JG, van Eijk, AM, ter Kuile, FO, et al, 2003: The effect of dual infection with HIV and malaria on pregnancy outcome in Western Kenya. AIDS17:585-9.

Bartoloni, A, Zammarchi, L, 2012: Clinical aspects of uncomplicated and severe malaria. Mediterr. J. Hematol. Infect. Dis. 4, 1:e2012026.

Beyrer, C, Karim, QA, 2013: The changing epidemiology of HIV in 2013. Curr. Opin. HIV AIDS 8, 4:306-10.

Bloland, PB, Wirima, JJ, Steketee, RW, et al, 1995: Maternal HIV infection and infant mortality in Malawi: Evidence for increased mortality due to placental malaria infection. AIDS 9: 721-6.

Braack, L, de Almeida, PGA, Cornel, AJ, Swanepoel, R, de Jager' C, 2018: Mosquito-borne arboviruses of African origin: review of key viruses and vectors. Parasit. Vectors 1:29-34.

Bronzan, RN, Taylor, TE, Mwenechanya, J, et al, 2007: Bacteremia in Malawian Children with Severe Malaria: Prevalence, Etiology, HIV Coinfection, and Outcome. J. Infect. Dis. 195: 895-9.

Butcher, GA, 2005: T-cell depletion and immunity to malaria in HIV-infections. Parasitology 130:141-4.

Byakika-Kibwika, P, Ddumba, E, Kamya, M, 2007: Effect of HIV-1 infection on malaria treatment outcome in Ugandan patients. Afr. Hlth. Sci. 7, 2:86-92.
Campell, J, Moore, D, Degerman, R, et al, 2009: Presented at the $16^{\text {th }}$ Annual Conference on Retroviruses and Opportunistic Infections, Montreal, Canada February 8-11th, abstract \# 33.

Cao, Y, Krogstad, P, Korber, BT, Koup, RA, Muldoon, M, et al, 1997: Maternal HIV-1 viral load and vertical transmission of infection: the Ariel Project for the prevention of HIV transmission from mother to infant. Nat. Med. 3, 5: 549-52.

CDC, 2019: Travelers' Health Update

Cohen, C, Karstaedt, A, Frean, J, et al, 2005: Increased prevalence of severe malaria in HIVinfected adults in South Africa. Clin. Infect. Dis. 41:1631-6.

Crampin, AC, Floyd, S, Glynn, JR, et al, 2002: Long-term follow-up of HIV-positive and HIV-negative individuals in rural Malawi. AIDS 16:1545-8.

Dondorp, A, Nosten, F, Stepniewska, K, et al, 2005: Artesunate versus quinine for treatment of severe falciparum malaria: a randomized trial. Lancet 366:717-20.

Enserink, M, 2014: After the windfall. Science 345, 6202:1258-9.

Francesconi, P, Fabiani, M, Dente, MG, et al, 2001: HIV, malaria parasites, and acute febrile episodes in Ugandan adults: A case-control study. AIDS 15:244-5.

French, N, Nakiyingi, J, Lugada, E, et al, 2001: Increasing rates of malarial fever with deteriorating immune status in HIV-1-infected Ugandan adults. AIDS 15:899-904.

Froebel, K, Howard, W, Schafer, JR, et al, 2004: Activation by malaria antigens renders mononuclear cells susceptible to HIV infection and re-activates replication of endogenous HIV in cells from HIV-infected adults. Parasite Immunol. 26:213-8.

German, P, Greenhouse, B, Coates, C, et al, 2007: Hepatotoxicity due to a drug interaction between amodiaquine plus artesunate and efavirenz. Clin. Infect. Dis. 44:889-92.

González, R, Ataíde, R, Naniche, D, Menéndez, C, Mayor, A, 2012: HIV and malaria interactions: where do we stand? Expert. Rev. Anti. Infect. Ther. 10, 2:153-65.

Green, MD, van Eijk, AM, van Ter, Kuile FO, et al, 2007: Pharmacokinetics of sulfadoxine-pyrimethamine in HIV-infected and uninfected pregnant women in Western Kenya. J. Infect. Dis. 196: 1403-12. 
Hamer, DH, Mwanakasale, V, Macleod, WB, et al, 2007: Two-dose versus monthly intermitent preventive treatment of malaria with sulfadoxine-pyrimethamine in HIV-seropositive pregnant Zambian Women. J. Infect. Dis. 196:158592.

Harris, C, Mills, R, Seager, E, Blackstock, S, Hiwa, T, 2018: Paediatric deaths in a tertiary government hospital setting, Malawi. Paediatr. Int. Child. Heth. 19:1-9.

Hewitt, K, Steketee, R, Mwapasa, V, et al, 2006: Interactions between HIV and malaria in non-pregnant adults: evidence and implications. AIDS 20:1993-8.

Hobbs, CV, Voza, T, Coppi, A, et al, 2009: HIV protease inhibitors inhibit the development of preerythrocytic-stage Plasmodium parasites. J. Infect. Dis. 199:134.

Hoffman, IF, Jere, CS, Taylor, TE, et al, 1999: The effect of Plasmodium falciparum malaria on HIV-1 RNA blood plasma concentration. AIDS 13:487-91.

Jaffar, S, Grant, AD, Whitworth, J, et al, 2004: The natural history of HIV-1 and HIV-2 infections in adults in Africa: a literature review. Bull WHO 82:462.

Johnstone, MJ, Crock, E, 2016: Transitions in nursing: Preparing for professional practice. In: Dealing with Ethical Issues in Nursing Practice. By E, Chang, J, Daly Sydney: Elsevier.

Kamya, M, Gasasira, A, Achan, J, et al, 2007: Effects of trimethoprim-sulfamethoxazole and insecticide-treated bednets on malaria in HIVinfected Ugandan children. AIDS 21:2059-66.

Kamya, MR, Gasasira, AF, Achan, J, et al, 2007: Effects of trimethoprim-sulfamethoxazole and insecticide-treated bed-nets on malaria among HIV-infected Ugandan children. AIDS 21: 2059-62.

Kamya, MR, Gasasira, AF, Yeka, A, Bakyait, N, Nsobya, SL, et al, 2006: Effect of HIV-1 infection on antimalarial treatment outcomes in Uganda: A population-based study. J. Infect. Dis. 193, 1:9-15.

Kaplan, JE, Benson, C, Holmes, KH, et al, 2009: Guidelines for prevention and treatment of opportunistic infections in HIV-infected adults and adolescents: Recommendations from CDC, the National Institutes of Health, and the HIV Medicine Association of the Infectious Diseases Society of America. MMWR. Recomm. Rep. 58: 1-9.

Karp, CL, Auwaerter, PG, 2007: Coinfection with HIV and tropical infectious diseases: Protozoal pathogens. Clin. Infect. Dis. 45:1208-12.

Kublin, JG, et al, 2005: Effect of Plasmodium falciparum malaria on concentration of HIV-1RNA in the blood of adults in rural Malawi: a prospective cohort study. Lancet 365: 233-5.

Laufer, MK, van Oosterhout, JJ, Thesing, $P$ C, et al, 2006: Impact of HIV-associated immuno-suppression on malaria infection and disease in Malawi. J. Infect. Dis. 193:872.

Lek-Uthai, U, Suwanarusk, R, Ruengweerayut, R, et al, 2008: Stronger activity of human immuno-deficiency virus type 1 protease inhibitors against clinical isolates of Plasmodium vivax than against those of $P$. falciparum. Antimicrob. Agents Chemother. 52:2435-40.

Marodi, L, 2006: Neonatal innate immunity to infectious agents. Infect. Immun. 74:1999-204.

Mermin, J, Ekwaru, JP, Liechty, CA, et al, 2006: Effect of co-trimoxazole prophylaxis, antiretro-viral therapy, and insecticide-treated bednets on the frequency of malaria in HIV-1infected adults in Uganda: A prospective cohort study. Lancet 367:1256.

Mermin, J, Lule, JR, Ekwaru, JP, 2006: Association between malaria and CD4 cell count decline among persons with HIV. J. Acquir. Immune Defic. Syndr. 41:129-32.

Miller, JM, Korenromp, EL, Nahlen, BL, W Steketee, R, 2007: Estimating the number of insecticide-treated nets required by African households to reach continent-wide malaria coverage targets. JAMA 297:2241-8.

Moro, L, Bardají, A, Macete, E, Barrios, D, Morales-Prieto, DM, et al, 2016: Placental microparticles and micrornas in pregnant women with Plasmodium falciparum or HIV Infection. PLoS One. Jan 12;11(1):e0146361

Morsy, TA, 2012: Insect bites and what is eating you? J. Egypt. Soc. Parasitol. 42, 2:291-308.

Mouala, C, Houze, S, Guiguet, M, et al, 2008: Imported malaria in HIV-infected patients enrolled in the ANRS CO4 FHDH study. J. Acquir. Immune Defic. Syndr. 49:55-60.

Mouala, C, Guiguet, M, Houze, S, et al, 2009: Impact of HIV infection on severity of imported malaria is restricted to patients with CD4 cell counts < 350 cells/microl. AIDS 23:1997-104. Mount, AM, Mwapasa, V, Elliott, SR, Beeson, JG, Tadesse, E, et al, 2004: Impairment of humoral immunity to $P$. falciparum mal-aria in pregnancy by HIV infection. Lancet 363: 1860. Muller, O, 1990: The clinical and parasitolog- 
ical presentation of Plasmodium falciparum malaria in Uganda is unaffected by HIV-1 in-fection. Trans. R. Soc. Trop. Med. Hyg. 84:336-9.

Neres, R, Marinho, CRF, Gonçalves, LA, Catarino, MB, Penha-Gonçalves, C, 2008: Pregnancy outcome and placenta pathology in Plasmodium berghei an infected mice reproduce the pathogenesis of severe malaria in pregnant pathogenesis of severe malaria in pregnant women. PLoS One. 3(2): e1608 doi: 10.1371/journal. pone. 0001608

Ogwang, R, Anguzu, R, Akun, P, Ningwa, A, Kayongo, E, et al, 2018: Asymptomatic malaria parasitaemia and seizure control in children with nodding syndrome; a cross-sectional study. BMJ Open. Oct 18;8(10):e023624. doi: 10.1136/bmj open-2018-023624.

Parise, M, Ayisi, J, Nahlen, B, et al, 1998: Efficacy of sulfadoxine-pyrimethamine for prevention of placental malaria in an area of Kenya with a high prevalence of malaria and human immunodeficiency virus infection. Am. J. Trop. Med. Hyg. 59:813-9.

Patnaik, P, Jere, CS, Miller, WC, et al, 2005: Effects of HIV-1 serostatus, HIV-1 RNA concentration, and $\mathrm{CD} 4$ cell count on the incidence of malaria infection in a cohort of adults in rural Malawi. J. Infect. Dis. 192:984-9.

Pereira, NR, Sarmento, A, Santos, L, 2015: Simultaneous diagnosis of severe imported Plasmodium falciparum malaria and HIV: report of three cases. Malar. J. Jul 10;14:266. doi: 10.1186/s12936-015-0780-6.

Qu, J, Zhou, T, Zhong, C, Deng, R, Lü, X, 2017: Comparison of clinical features and prognostic factors in HIV-negative adults with cryptococcal meningitis and tuberculous meningitis: a retrospective study. BMC Infect. Dis. Jan 10; 17(1):51. doi: 10.1186/s12879-016-2126-6.

Quinn, TC, Wawer, MH, Swankambo, N, et al, 2000: Viral load and heterosexual transmission of the human immunodeficiency virus type 1. N. Engl. J. Med. 342:921-7.

Robb, ML, Eller, LA, Kibuuka, H, Rono, K, Maganga. L, et al, 2016: Prospective Study of Acute HIV-1 Infection in Adults in East Africa and Thailand. N. Engl. J. Med. 374, 22:2120-30. Rosenthal, PJ, 2008: Artesunate for the treatment of severe falciparum malaria. N. Engl. J. Med. 358:1829-33.
Santoro, MM, Perno, CF, 2013: HIV-1 Genetic Variability and Clinical Implications. ISRN Microbiol. Published online 2013 Jun 17. doi: 10.1155/2013/481314.

Schultz, LJ, Steketee, RW, Macheso, A, et al, 1994: The efficacy of antimalarial regimens containing sulfadoxin-pyrimethamine and/or cholorquine in preventing peripheral and placental $\mathrm{Pl}$ asmoidum falciparum infection among pregnant women in Malawi. Am. J. Trop. Med. Hyg. 51: 515-9.

Shah, S, Smith, E, Obony, C, et al, 2006: HIV Immunosuppression and antimalarial efficacy: sulfadoxine-pyrimethamine for the treatment of uncomplicated malaria in HIV-infected adults in Siaya, Kenya. J. Infect. Dis. 194: 1519-22.

ter Kuile, FO, Parise, ME, Verhoeff, FH, Udhayakumar, V, Newman, RD, et al, 2004: The burden of co-infection with human immunodeficiency virus type 1 and malaria in pregnant women in Sub-Saharan Africa. Am. J. Trop. Med. Hyg. 71:41-8.

Ter Kuile, FO, Steketee, RW, 2007: Intermittent preventive therapy with sulfadoxine-pyrimethamine during pregnancy: Seeking information on optimal dosing frequency. J. Infect. Dis. 196: 1574-80.

Thera, MA, Sehdev, PS, Coulibaly, D, et al, 2005: Impact of trimethoprim-sulfamethoxazole prophylaxis on falciparum malaria infection and disease. J. Infect. Dis. 192:1823-6.

Ticconi, C, Mapfumo, M, Dorrucci, M, Naha, N, Tarira, E, 2003: Effect of maternal HIV and malaria infection on pregnancy and perinatal outcome in Zimbabwe. J. Acquir. Immune Defic. Syndr. 34, 3:289-94

Tilley, D, Crock, E, 2016: HIV Management For Nurses and Midwifes (OASHM 2016, ashm hiv management in Australasia: Supporting the HIV, viral hepatitis and sexual health workforce. Van Geertruyden, JP, Mulenga, M, Mwananyanda, L, et al, 2006: HIV-1 immune suppression and antimalarial treatment outcome in $\mathrm{Za}$ mbian adults with uncomplicated malaria. J. Infect. Dis. 194:917-20.

WHO, 2004: A strategic framework for malaria prevention and control during pregnancy in the African region. Brazzaville: WHO Regional Office for Africa. 